\title{
Knowledge, action and reflection: A theoretically based model for use with high fidelity simulation in nursing education
}

\author{
Mary J ess Regan, Rachel Onello \\ School of Nursing, University of Maryland, Baltimore, USA \\ Correspondence: Mary Jess Regan. Address: 655 West Lombard Street, Room 475D, Baltimore, MD 21201, USA. Email: \\ regan@son.umaryland.edu.
}

Received: August 15, 2012

DOI : $10.5430 /$ jnep.v3n7p106

Accepted: September 25, 2012

Online Published: January 15, 2013

\section{Abstract}

The lack of direction and sound empirical evidence to support the use of high fidelity simulation in nursing education has been attributed to the lack theoretically based models that can be used to structure the use of educational simulation. This paper proposes a theoretically-based model called the Knowledge, Action and Reflection (KAR) model that can be used to structure high fidelity simulations. Theories from nursing, education and cognitive psychology were reviewed and synthesized into a comprehensive model that is designed to help educators design, implement, and evaluate high fidelity simulation scenarios for nursing education. The use of a theoretically defined model provides a testable framework to build evidence necessary to identify best practices for educational simulation.

\section{Key words}

High fidelity simulation, Nursing education, Theoretical model, Simulation design, Clinical reasoning

\section{I ntroduction}

Despite the increase in the use of educational simulation the evidence about its effectiveness as an educational strategy is inconsistent. The lack of sound empirical evidence is attributed to an absence of theoretically based models to shape the use of educational simulation ${ }^{[1]}$. Theory organizes knowledge, guides practice, and facilitates the discovery of new innovations that in turn advance practice ${ }^{[2]}$. A theory based conceptual model for simulation could be used to guide the design and implementation of clinical scenarios and provide measurable learning outcomes. Without a theory based conceptual model, the technology involved in high fidelity simulation can influence the way simulation is used ${ }^{[1]}$. This can result in a mismatch between desired learning outcomes, the level of technology that is used, and evaluation of learning. Consequently, the application of simulation is often "piecemeal" and lacks a "comprehensive approach" necessary for evaluation of the findings ${ }^{[1, \mathrm{p} .392]}$. This is reflected in the systematic reviews that showed studies about simulation used inconsistent and informal evaluation tools that made it impossible to compare and synthesize the findings ${ }^{[3-5]}$. One of the reviews found that $90 \%$ of simulation studies did not theory at all or used it insufficiently ${ }^{[5]}$. 
In fact, many authors do cite using theory to frame their approach to educational simulation. Learning theories such as Dewey's Theory of Experiential Learning or Kolb’s Cycle of Learning are commonly used as the basis for educational simulation ${ }^{[6]}$. However, these broad theories lack specificity and therefore lack ready measurement of learning outcomes. For theory to serve effectively as a conceptual model for simulation it needs to be comprehensive enough to cover the depth and breadth of the domain of nursing knowledge including the knowledge, practices and scope of the discipline embedded in discrete specialties ${ }^{[7]}$. Single theories are generally too broad to provide distinct testable outcomes that reflect the multiple facets that combine to form the discipline. Similarly, no single mid-range level theory is broad enough to capture the full breadth of nursing practice. Therefore it is necessary to integrate multiple theories into a model for educational simulation that provide the specificity needed to measure discrete attributes of clinical practice while providing an accessible framework for learning processes and outcomes.

Educational simulation is currently emerging as a viable alternative to supplement to clinical education, particularly for teaching clinical reasoning ${ }^{[8-11]}$. As a result, developing observable indictors for measuring clinical reasoning and decision-making is imperative. Construction of knowledge is the essential component needed to teach students how to make sound clinical judgments. Knowledge used to shape action in a clinical situation is constructed by selecting different types of information that are contextually applicable and also by drawing on past experience ${ }^{[12]}$. Purposively teaching students how to construct knowledge requires a learning paradigm. Currently there is evidence to suggest that in nursing, educational simulation is framed by a teaching model typified by practices aimed at "transferring knowledge from faculty

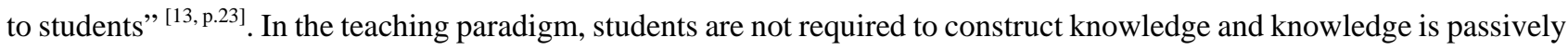
transferred to the learner. To teach students how to think clinically, it is necessary to use a learning paradigm because it actively engages them in the process of constructing knowledge and critiquing the attitudes and beliefs that motivate their clinical judgments and action. As such, incorporating the development of clinical judgment and reasoning as a direct outcome of simulation should be reflected in any framework meant to successfully guide the use of educational simulation.

This paper presents a theoretically based conceptual model that can be used to guide the design, implementation and evaluation of educational simulation for nursing education. The Knowledge-Action-Reflection (KAR) model is designed to promote three essential components of clinical reasoning, namely Knowledge attainment and interpretation, clinical Action, and Reflection in and on action. The conceptual model subsumes the central tenets of Jeffrie's Nurses Education Simulation Framework (NESF) with its focus on simulation as a pedagogical tool to promote student and instructor outcomes ${ }^{[14]}$. Multiple learning and cognitive theories are nested in Mezirow's transformative learning theory that is the predominant theoretical approach. Tanner's framework for clinical reasoning in nursing to validate students' cognitive processes of decision-making ${ }^{[12]}$. In addition, Ackoff's hierarchy of knowledge management is used to structure how students are taught to systematically develop clinical understanding from data ${ }^{[15]}$. Finally, Liaschenko and Fischer's knowledge taxonomy guides the students to identify different forms of knowledge from the patient record. We present the model here for consideration for use with educational simulation in nursing. Each aspect of and its relationship to the model as a whole is described below.

\section{The KAR conceptual model for educational simulation}

The KAR model is a standardized framework for educational simulations designed to promote evidence-based clinical decision-making among students by facilitating the development of clinical reasoning skills. It can be used to develop a core set of high fidelity simulations based on evidence-based and best-practice guidelines or applied to simulations focused on developing and demonstrating skill mastery. In addition the model provides a format for testing learner competence, and remediating students who encounter difficulties with required core competencies. The theories underpinning the KAR model emphasize the cognitive processes involved in processing complex information and translating knowledge into appropriate action. The intent is to help students learn how to access and interpret different types of data and convert that into actionable knowledge about the clinical case and the person at the heart of the encounter. Central to the process is the reliance on reflection to transform attitudes and beliefs of the learner related to knowledge attainment, 
interpretation and application to a clinical situation. This feature is intended to teach students how to think in meaningful ways that will help them construct knowledge necessary to guide their clinical actions and provide holistic evidence based care. While for the purposes of this publication the model is presented in a linear fashion, in practice the processes are used iteratively. The basic assumption underlying the KAR model is that educational simulation is a technique, rather than simply a technology, that generates applied clinical understanding, knowledge, and wisdom.

The theoretical model integrates multiple theoretical approaches into each distinct component in order to provide testable outcomes. Transformative learning theory is the overarching educational approach. The central tenet of transformative learning is to teach learners to think autonomously "in a collaborative context rather than uncritically act on the received

ideas and judgments of others" ${ }^{[16, p .8]}$. In this theory, autonomous thinking is defined as the ability to critically reflect on ones attitudes and beliefs and to validate assumptions against the experience of peers. The theory is designed to transform the learners' frames of reference. Frames of reference are constituted by two dimensions namely habits of mind and points of view. Habits of mind are the broad orienting beliefs that motivate action and are assimilated over the course of a lifetime. An example of a habit of mind is ethnocentricity or ageism. Habits of mind are usually subliminal cognitive frames of reference and are therefore difficult to access. Points of view are the second dimension. Points of view originate from habits of mind and orient the individual toward action or understanding in a particular situation. For example if a nurse holds a habit of mind related to ageism, then the care provided will be influenced by that cognitive frame of reference. Points of view are constantly changing in response to life experience and reflection and readily accessible by the individual. Similarly, Brookfield's approach to critical reflection is used to shape review and transformation of points of view. Detail about the application of is provided in the relevant sections below.

Transformative learning theory is appropriate to promote robust clinical reasoning because it provides the opportunity for reflective discourse and structured review of real life experience. As a result, the learner is guided to critically assess the rationale for action in clinical situations and imagine alternative trajectories of action. Transformative learning theory also builds strong team skills essential to nursing practice by placing learning in the context of group learning and problem solving.

\section{Component one: Knowledge attainment, interpretation and application}

Knowledge attainment, interpretation and application are skills that are at the root of clinical competence. The focus on building those skills is a unique feature of this model and the innovation comes from the design template that extends beyond the high fidelity simulation to encompass how and what data are used to inform clinical action. Knowledge attainment, interpretation and application are a central feature of the KAR model that is intended to teach students how to think in meaningful ways that will help them construct the necessary knowledge to guide their clinical actions. Activities, such as educational simulation, can be used to force learners to think about what is needed in a given situation, as well as training their evidence-based patient-centered care. Often, simulation is used for the later purpose - namely to train providers' responses to high acuity events that occur rarely in clinical practice such as cardiac arrest. This can be problematic because it doesn't train the learner to habitually seek out and integrate research findings into practice ${ }^{[17]}$. The KAR model is purposively designed to build student's clinical reasoning since it is the cognitive process that nurses use to review and analyze data for the purpose of making decisions about care ${ }^{[18]}$. The processes used in the KAR model are intended to reform students' cognitive processes so they routinely integrate best practices shaped by the patient's unique beliefs, values, wants and desires, in order to produce practitioners whose lifelong practice is patient-centered and evidence-based. The KAR model can be used to leverage this attribute of simulation to train students' cognitive processes so that they habitually rely on up-to-date evidence-based resources rather than memorized content that may be out of date or inaccurate. Learning activities can be designed that will develop students' cognitive process based on Ackoff's hierarchy of knowledge management ${ }^{[15]}$. Ackoff asserts that knowledge is a product of combining raw data to form information that defines what can be known. Data has little inherent value unless is it combined into meaningful bytes. For example, the numbers 160 and 100 mean nothing until they are combined into a blood pressure reading - then they become information. When bytes of information are aggregated they create knowledge that can be used to know how to act. In the 
previous example of a blood pressure the aggregated bytes could be factors such as patient history and current symptoms that would provide a context for interpreting the finding.

In nursing, students need to learn what types of data they need to guide the care they provide. In the KAR model, Liaschenko and Fischer's knowledge taxonomy is used to define the types of knowledge that students are systematically taught to access, review and analyze. According to Liaschenko and Fischer, nurses rely on three types of knowledge to inform their practice ${ }^{[19]}$. This includes case knowledge that is things such as the diagnosis, physiology, pathology, pharmacology and treatment options. The second form of knowledge is patient knowledge. Patient knowledge includes elements that are specific to the person such as age, gender, demographics or marital status. This knowledge is necessary to understand or predict the individual's response to treatment. The last source of knowledge is person knowledge that that is unique to the individual and relates to beliefs, values and preferences - the context of their existence. This is different from patient knowledge because it directs the nurse toward knowing what is important for a particular person based on their lived experience and value system. Liaschenko and Fisher describe this as "knowing how the person is situated in and engages with the world” ${ }^{[19, \text { p.27] }}$. The knowledge taxonomy is used to guide the types of data students access and structures how they combine that into information. This is done using a Clinical Reasoning Worksheet (CRW). The CRW is unique to the KAR model and is designed to teach and test students' ability to select and sort relevant case data and combine it into meaningful informational structures that in turn generate clinically relevant knowledge to define the clinical actions that are needed. Students complete a CRW prior to engaging in a high fidelity simulation. The exercise guides them to gather data from the health record in order to identify the core health issues and define priorities of care. The central purpose is to promote thinking about the patient as a person with unique needs as well as training them to prioritize the clinical issues based on the available evidence and the physiological and psychosocial rationale. The CRW provides a mechanism for the student to learn where information is within the health record as well as identify information that is needed to select practice options. It structures data gathering of pertinent case and patient knowledge and any person knowledge represented in the chart. Knowledge attainment continues throughout the simulation and is represented in different forms or inquiry based on where the knowledge is most accessible. For example, the chart is usually a good source of patient knowledge and some case knowledge, while the internet, textbooks and clinical practice guidelines are all excellent resources for case knowledge, and person knowledge is best attained from interviewing the patient.

Learners are provided the health record and the CRW in advance of the HFS based on their progress in the curriculum. For example, students in the first semester of the nursing program may be given the material with more preparatory time than a final semester student. This is done to allow for optimal learning because it provides enough time for them to access and synthesize the knowledge without the added element of time pressure. Gonzalez demonstrated that time constraints negatively impact decision-making and subsequent actions because the time pressure influences the cognitive and problem solving ability in uncertain situations ${ }^{[20]}$. This is particularly relevant to educational simulation because it suggests that in order to optimize the learning that can be achieved, the element of time pressure must be minimized. In the KAR model, de-indentified health records of actual health encounters are used to build scenarios. Each chart contains the appropriate reports, health history, assessments and care documentation. This is to ensure that the cases are authentic and reflect the reality of practice. To complete the CRW exercise, students assess the record and determine what the health issues are and define the priorities of care. The exercise requires the student to explain why they prioritize the issue based on the available evidence and the physiological and psychosocial rationale.

The CRW provides evidence of measurable outcomes namely, information literacy, identification of case, patient and person knowledge, linkages between the underlying human physiology and patient presentation and prioritization of clinical actions. Since this model is theory driven, the outcomes are derived from the theoretical concepts. For example, evidence of information literacy would be found in the types of reference material a learner cites using. Similarly, person knowledge is evidenced by inclusion of contextual material in the clinical priorities and patient specific care. Moreover, outcomes related to the students' cognitive processes are based on Ackoff's hierarchy of knowledge management ${ }^{[15]}$. These outcomes relate to students' ability to sort and select relevant case data and combine it into meaningful 
informational structures that in turn generate clinically relevant knowledge. Finally, the model is designed to promote evidence-based clinical decision-making. A primary goal is to leverage simulation to train student's cognitive processes so that they habitually rely on up-to-date evidence-based resources rather than memorized content that may be out of date or inaccurate.

\section{Component two: Clinical Action}

Tanner's framework for clinical reasoning in nursing provides a structure to guide and evaluate the efficacy of students' cognitive processes during the high fidelity simulation (HFS) ${ }^{[12]}$. The action component of the KAR model is a direct application of their knowledge and cognitive processing and provides tangible and measurable evidence of what a student can do that also demonstrates what they know ${ }^{[21]}$. Outcomes-based student evaluation tools are used to test the effectiveness of students' demonstrated psychomotor, cognitive and affective domain knowledge ${ }^{[22]}$. Each HFS is comprised of four discrete but integrated components - communication, assessment, clinical prioritization and clinical action. Criterion based instruments are used to measure student performance in the HFS. Each instrument is based on the attributes of clinical proficiency that are determined by the objective of the simulation. Each discrete nursing function is accompanied by a set of expected actions. For example, if the simulation involves giving a medication then the measure of proficiency is the degree to which the learner correctly completes all the required steps of the action. In addition in the KAR model, student action is reviewed for inclusion of evidenced-based patient-centered care.

Based on the course objectives, a de-identified health record that represents a relevant clinical situation is selected. Available evidence and best practices related to the required care are isolated and used to define what optimal practice should be in the given situation. The level of the course within the curriculum determines the degree of complexity in terms of what actions are deemed essential, preferred or optional. These data are used to create a storyboard to plot out the expected student actions and the correlating human patient simulator (HPS) responses. Content experts validate the scenarios and the simulation is beta tested to allow the faculty to correct any problems that are identified. Key features of the HPS such as hair, clothing and even skin color are changed to suit the specifics of the case and to create a realistic environment.

The high fidelity simulation starts with the student receiving report. The KAR model is specifically designed to teach and evaluate students' communication skills on multiple levels. For this reason, each high fidelity scenario includes, depending on the case, either an oral, taped or written report. As in the clinical setting, the report contains progress information about the patient and in some cases lays out ongoing care needs. Based on the cues contained in the report the student can revise the clinical priorities they identified.

The design characteristics of the high fidelity simulation scenarios themselves are also very different than normal when the KAR model is used to structure the design. To begin with, they are focused on teaching or evaluating a very specific set of competencies that are well defined and clearly measurable. The time required for each scenario is based on the learning outcomes. Therefore scenarios that are focused on a small set of competencies can be completed quickly - typically in less than 10 minutes, but the model can also be used for immersive learning experiences that replicate an entire shift. The HPS responses are not programmed to allow flexibility in terms of the how the simulators respond to the student actions. When the student's responses are not aligned with the preferred actions, the person operating the simulator provides cues to guide the student to act in specific ways. The level of the course moderates this feature; in a final semester course the cues would be more diffuse and less directive than in a first semester course. The aim is for students to know clearly what is needed and to act in an appropriate way. A standard design feature is that all the scenarios include a 'confederate' who plays the role of a family member, friend or other non health care related individual. The use of confederates is intended to provide opportunities for students to become accustomed to dealing with this eventuality in practice. Encountering others aside from the patient in high fidelity simulation allows students to experience the need to deal with and effectively interact with individuals aside from the patient. At the completion of the clinical action segment of the high fidelity scenario, students are required to communicate their findings in some way. This may be requesting a physician order, delegating care, 
requesting help, providing an explanation to the patient or a family member, or documenting the encounter. This activity is the starting point for debriefing.

\section{Component three: Reflection on Action}

The debriefing process used in the KAR model is a synthesis of the principles in Rudolph and colleagues' Debriefing with Good Judgment approach, Harvard Center for Medical Simulation's Debriefing Assessment for Simulation in Healthcare (2010 [DASH]) and Brookfield's framework for critical reflection ${ }^{[23,24]}$. The debriefing starts with the instructor prompting the student who completed the high fidelity simulation to articulate what they would do next and how they would achieve their plan of action. The debriefing guides the student to reflect on their clinical action in light of their interpretation of the clinical data and priorities of care. Evaluation of the degree to which the clinical actions were evidence-based best practices is completed to allow the student to build clinical wisdom about how (and why) they should act in similar situations in the future. In line with Brookfield, emphasis in the KAR model is placed on guiding the reflective process to include review of assumptions and beliefs embedded in the clinical actions. Assumptions motivate human actions by influencing the belief system. Critical review of assumptions is an essential component of critical reflection and because they are subliminal cognitive structures, they are not readily amenable for review. However, assumptions are frequently expressed in human actions and therefore with adequate inquiry people can be guided to reveal them. Similarly, the debriefing process is designed specifically to provide a mechanism to make apparent the assumptions that influence how data are interpreted and demonstrate how this cognitive activity is fundamental to sound evidencebased clinical decision-making.

Eliciting assumptions and frames of reference is facilitated by the advocacy - inquiry method inherent in the debriefing with good judgment approach. The advocacy involves the facilitator stating an observation of an action or event that they noted during the high fidelity simulation and what the instructor thinks about it. This could be something like "I noticed during the simulation that you went to the orders before applying the oxygen to the patient. I'm concerned because the patient's oxygen saturation was $81 \%$ and our protocol is to apply oxygen and then notify the provider.” This is followed by an open-ended inquiry that is designed to elicit the student's perspective in relation to their actions. For example, "can you walk me through what you were thinking at the time"? This approach helps to bring the frames of reference to the forefront and also guides the student to think critically about their actions and why they act in certain ways. In addition, it enables the instructor to understand the student's frame of reference that increases the opportunity for them to provide individualized teaching shaped by the student's specific needs. Finally, because the approach emphasizes respect and mutual understanding, it minimizes the need for defensiveness or embarrassment on the part of the student to optimize learning.

The advocacy - inquiry method is paired with the six key element of debriefing delineated in the DASH model. The DASH model is intended to ensure that the instructor is employing best practices to facilitate learning. The six key elements are:

1) Establishing an engaging learning environment;

2) Maintaining an engaging learning environment;

3) Structuring the debriefing in an organized way;

4) Provoking engaging discussions;

5) Identifying and exploring performance gaps;

6) Helping trainees achieve or sustain good future performance.

Instructors establish an engaging learning environment by clarifying the learning objectives, roles and expectations. The focus of this first element is to convey a commitment to respecting learners and their perspective. Another dimension of 
the element is aimed at establishing a "fiction contract". A fiction contact is where the instructor and student acknowledge that while the scenario is not entirely realistic, they agree to treat it as such. The second element, maintaining an engaging learning environment, is an extension of the first element. It is intended to ensure that the instructor maintains the respectful and psychologically safe learning environment. The third element addresses the organization and flow of the debriefing. Learners are actively encouraged to express their emotions and reactions to the scenario before the critical reflection begins. The advocacy-inquiry method by the facilitator guides the learner's analysis of their performance and helps them apply the knowledge they gain to future practice. At the end of this phase, learners summarize what they have learned during the process in order to reinforce the learning that has occurred. The process is designed to allow students to make meaning of their experience in ways that promote perspective transformation to make the learners more inclusive, discriminating and open ${ }^{[25]}$. For the fourth element, the debriefer provokes engaging discussions using the advocacyinquiry method while paying careful attention to verbal and non-verbal cues of both the debriefer and learner that facilitate or inhibit discussion. Recognizing and addressing the disengaged or upset learner in a constructive way is also critical to maintaining engaging discussions. The fifth element focuses on identifying and exploring gaps in the learner's performance. To achieve this, the debriefer provides feedback to facilitate the learner's clarity about what they did well and how they can improve in the future. Positive affective behaviors are required to assist the learner to move beyond what they did to why they did it and to encourage them to reflect on what they were thinking at the time. Helping learners achieve or sustain good performance in the future is the final element of the DASH model. It is intended to reduce the performance gaps through critical discourse, congruent with transformative learning theory. It is essential that the debriefer has expertise in the topic in order to share evidence-based best practice applicable to the learning outcomes of the scenario.

Perspective transformation is relevant not only for the learner but also provides a mechanism for the debriefer to refine his or her debriefing skills. Therefore, it is an important feature of the KAR model that the students have an opportunity to evaluate the effectiveness of the debriefing. The DASH model includes a valid and reliable student-rater tool based on the six elements of good debriefing practices that students can use to evaluate the debriefer. The KAR model incorporates this tool into the evaluative process to help solidify learner reflection on the debriefing process as well as to provide constructive feedback for the debriefer.

\section{Conclusion}

The KAR model synthesizes multiple relevant theories into a comprehensive whole that is intended to guide the design, implementation, and evaluation of high fidelity simulation in nursing education. Use of the model has implications for educating practitioners who inherently understand how to access, evaluate and apply data in ways that goes beyond current approaches. In addition, practitioners exposed to this model will be trained to routinely critique their own cognitive perspectives and critically analyze clinical situations.

The theoretical base for the model also provides robust measures related to learner's skills and abilities. This has significant implications for research about the use of HFS and provides a robust framework to conduct research needed to build an evidence basis for the use of this educational technology. In the process of using the model for research we fully expect that it will be refined and evolved in order to adapt to the needs of students and educators alike.

\section{References}

[1] Schiavenato M. Reevaluating simulation in nursing education: Beyond the human patient simulator. J Nurs Ed. 2009 ; 48 : 388-394.

[2] Walker L, Avant K. Strategies for theory construction in nursing. New Jersey: Pearson Education, 2005.

[3] Cant R, \& Cooper S. Simulation-based learning in nurse education: systematic review. J Adv Nurs. 2010; 66: 3-15. PMid:20423432 http://dx.doi.org/10.1111/j.1365-2648.2009.05240.x 
[4] Harder B. Use of simulation in teaching and learning in Health Sciences: A systematic review. J Nur Educ. 2010 ; 49 : 23-28. PMid:19731886 http://dx.doi.org/10.3928/01484834-20090828-08

[5] Rourke L, Schmidt M, Garga, N. Theory-based research of high fidelity simulation use in nursing education: a review of the literature. Int J Nurs Educ Schol. 2010; 7: 1-11. PMid:20361859 http://dx.doi.org/10.2202/1548-923X.1965

[6] Overstreet M. The use of simulation technology in the education of nursing students. Nurs Clin North Am. 2008 ; 43 : 593-603. PMid:18940416 http://dx.doi.org/10.1016/j.cnur.2008.06.009

[7] Meleis A. Theoretical nursing: Development and progress. Pennsylvania: J.B. Lippincott, 1991.

[8] American Association of Colleges of Nursing. The essentials of baccalaureate education for professional nursing practice. 2008. Available from: http://www.aacn.nche.edu/Education/pdf/BaccEssentials08.pdf.

[9] Agency for Healthcare Research and Quality. Improving Patient Safety Through Simulation Research. 2008. Available from: http://www.ahrq.gov/qual/simulproj.htm

[10] Kuiper R, Heinrich C, Matthias A, Graham M, Bell-Kotwass L. Debriefing with the OPT model of clinical reasoning during high fidelity patient simulation. Int J Nurs Educ Schol. 2008; 5: 1-14. PMid:18454731 http://dx.doi.org/10.2202/1548-923X.1466

[11] Nehring W. U.S. boards of nursing the use of high-fidelity simulators in nursing education. J of Prof Nurs. 2008; $24: 109-117$. PMid:18358446 http://dx.doi.org/10.1016/j.profnurs.2007.06.027

[12] Tanner C. Thinking like a nurse: a research-based model of clinical judgment. J of Nurs Educ. 2006; 45; 204-211. PMid:16780008

[13] Barr R., Tagg J. From teaching to learning: A new paradigm for undergraduate education. Change. 1995; $27:$ 12-25. http://dx.doi.org/10.1080/00091383.1995.10544672

[14] Jeffries P. Simulation in nursing education: From conceptualization to evaluation. New York: National League of Nursing Publishing, 2007.

[15] Ackoff R. From Data to Wisdom. J of Applied Sys Anal. 1989; 16; 3-9.

[16] Mezirow J. Transformative learning: Theory to practice. New Dir for Adult Cont Educ. 1997; 74: 5-12. http://dx.doi.org/10.1002/ace.7401

[17] Day L. Simulation and the teaching and learning of practice in critical care units. Amer J of Crit Care. 2007 ; 16 : 504-507. PMid:17724248

[18] Fisher A, Fonteyn M. An exploration of an innovative methodological approach for examining nurses' heuristic use in clinical practice... including commentary by Graves JR. Schol Inq Nurs Pract. 1995; 9; 263-279. PMid:8570978

[19] Liaschenko J, Fisher A. Theorizing the knowledge nurses use in the conduct of their work. Schol Inq Nurs Pract. 1999 ; $13: 29-41$. PMid:10420795

[20] Gonzalez C. Learning to make decisions in dynamic environments: Effective of time restraints and cognitive abilities. J of Hum Fac Erg Soc. 2004; 46: 449-460. http://dx.doi.org/10.1518/hfes.46.3.449.50395

[21] del Bueno D, Weeks L, Brown-Stewart P. Clinical assessment centers: a cost-effective alternative for competency development. Nurs Econ. 1987; 5: 21-26. PMid:3100966

[22] Redman R, Lenburg C, Hinton Walker P. Competency Assessment: Methods for Development and Implementation in Nursing Education. Online J of Iss in Nur. 1999; 4.

[23] Rudolph J, Simon R, Dufresne R, Raemer D. There’s no such thing as “nonjudgmental” debriefing: A Theory and method for debriefing with good judgment. Sim Hlthc. 2006; 1: 49-55.

[24] Brookfield S. Becoming a critically reflective teacher. California: Jossey Bass, 1995.

[25] Mezirow J. Learning as transformation: Critical perspectives on a theory in progress. California: Jossey Bass, 2001. 